\title{
Strategic Research on B2B E-commerce based on the Vertical Industry
}

\author{
Liu Bo
}

China West Normal University, Institute of foreign languages, Sichuan, Nanchong, China

Key Words: e-commerce, strategic management, key factor model, fuzzy comprehensive evaluation method

\begin{abstract}
At present we have the insufficient strategic research on B2B e-commerce company in the vertical industry. In the practical application there are more problems about the existing e-commerce strategy research. We carry out the research on strategy scheme evaluation of B2B e-commerce in the vertical industry based on AHP and fuzzy evaluation to build the strategic fuzzy mathematical comprehensive evaluation model of B2B e-commerce company in the vertical industry in the paper. We choose the optimal strategy scheme from the alternative strategy schemes, and then we make an empirical analysis. Finally, we put forward some suggestions and prospects for the future researches on the basis of the whole paper.
\end{abstract}

\section{Introduction}

Because the historical time scale of the strategic research on B2B e-commerce is relatively short, the environment which we have to face is complex and changeful. It is full of uncertainties. What's more, it is relatively difficult for us to obtain the environmental information. Therefore, we should pay attention to the analysis of the strategic environmental factors. The main object of external environment analysis is the environment related with the strategy. That is to say, there are some external factors which have a direct and significant impact on B2B e-commerce company in the vertical industry. It usually includes some aspects, such as policy, economy and technology, etc. This is so-called PEST analysis method. In the strategic research, for B2B e-commerce development in the vertical industry, some factors have the promoting and incentive function, and some factors have the obstructive and restrictive function. These factors are related to each other. And there are different influences on the future development in size, speed, and other aspects.

\section{Analysis of B2B E-commerce Internal Environment in Vertical Industry}

1. B2B E-commerce External Key Factors Model in Vertical Industry

After we analyze the value chain of the B2B e-commerce between the external environment and company interior in vertical industry, we can establish B2B e-commerce external factors evaluation matrix in vertical industry. The main steps of establishing external factors evaluation model are shown as follows.

(1)We list the key external environment factors which the B2B e-commerce company in the vertical industry faces. In the external factor evaluation model, the listed key external factors generally should be controlled between $10 \sim 15$. Here we choose 10 representative key factors.

(2)We determine a weight for each factor. The weight should be the value between 0 . 0 (not important)and 1. 0(very important). The weight of each factor shows that this factor has the relative importance for the enterprise's success in an industry. The important factor has the big weight, and the unimportant factor has the small weight. The sum of each factor should equal 1.

(3)According to the actual characteristics of industry development, we give each key factor a 
score so as to show that this factor is the great threat (-2 score), the slight threat ( -1 score), the general opportunity (1 score), or the big opportunity (2 score).

(4)We multiply the weight of each factor by the score given by us so as to obtain the corresponding weighted score of this key factor.

(5)We add up the weighted score of each factor, and the final sum is an enterprise's total weighted score.

At the same time, no matter how many important opportunities or threats are included by this model, the enterprise's total weighted score has the highest score, namely 2 scores, and the lowest score, namely -2 scores, and the average number is 0 score. The enterprises whose total weighted scores are higher than 0 score are in the attractive industry. What's more, there are many external opportunities. On the contrary, the enterprises whose total weighted scores are lower than 0 score are in the weak attractive industry. So they face more external threats.

This is the external key factor model routine table of B2B e-commerce in vertical industry. The establishment of the routine table is a reflection of the above model. All the data are from the strategic data analysis on B2B e-commerce company in certain vertical industry. Among them, the opportunity index sum in the following table is 0.703 , and the threat index sum is -0.818 .

2.Weight Determination with the Improved Analytic Hierarchy Process

Analytic hierarchy process is a kind of multicriteria decision process. It provides a kind of basic method of representing decision factors measurement. This method takes the form of relative scale, and makes full use of the person's experience and judgment ability. Under the hierarchical structure, according to certain rules and relative proportion scale with much regulation it depends on the policymakers' judgments, and makes the relative importance of related factors with the same level be compared in pairs, and synthesizes the scheme from top to bottom according to the level to measure the decision objectives. This measurement is expressed with relative importance weights in the end. The final result of analytic hierarchy process unifies many factors about the tangible and intangible, quantitative and qualitative factors. It not only can be used as a decision-making basis but also can be used as the important means which can solve many problems with the social and economic systems.

The basic thought: We set the factors considered by us are $A_{1}, A_{2}, \ldots, A_{n}$, then we use the three scale method to determine the comparison matrix. It is

$$
C=\left[\begin{array}{lll}
c_{11} & C_{12} \cdots & c_{1 n} \\
C_{21} & C_{22} \cdots & C_{2 n} \\
\vdots & \vdots & \vdots \\
C_{n 1} & C_{n 2} \cdots & C_{n n}
\end{array}\right]
$$

Among them, the comparison matrix $C$ has the following features.

The $i$ th element is more important than the $j$ th element.

$\left\{c_{i j}=1\right.$ The $i$ th element is the same important with the $j$ th element. $(i, j=1,2,3, \ldots, \mathrm{n})$

0 The $i$ th element is less important than the $j$ th element.

And $c_{i i}=1(i=1,2, \ldots, n)$, that is to say, the comparative result of element itself is 1 , and it shows that the numerical value on the main diagonal is 1 in the comparison matrix.

Among them, $r_{i}=\sum_{j=1}^{n} c_{i j}(i=1,2, \ldots, n)$ 
It shows the sorting index of importance about each key factor.

\section{Evaluation Selection of B2B E-commerce Company Strategy Scheme in Vertical Industry}

We use the principle and method of the mentioned fuzzy mathematics. The author sets up a comprehensive evaluation model of B2B e-commerce company strategy in vertical industry with strong generality. So it is convenient and feasible, and correct and reliable. And it has the comprehensive evaluation results.

$\mathrm{U}=\left(\mathrm{u}_{1}, \mathrm{u}_{2}, \ldots, \mathrm{u}_{\mathrm{m}}\right)$ is an index set which is made up of evaluation index. $\mathrm{U}_{\mathrm{i}}(\mathrm{i}=1,2, \ldots, \mathrm{m})$ represents the last level indicator of evaluation index system. We make B2B e-commerce company's overall development strategy in vertical industry be divided into four levels.

(1)The strategic general objective: g

(2)The specific strategic goal: $g_{1} g_{2} \ldots, g_{n}$

(3)The measures guarantee: $c_{1} c_{2} \ldots, c_{m}$

(4)The specific index: $d_{1} \quad d_{2} \ldots, d_{m}$

We set $A=\left(A_{1}, A_{2}, \ldots, A_{m}\right)$ as a weight set. $A_{i} \geq 0, i=1,2, \ldots, m$ represents the weight of the ith index in the index set $U, \Sigma A_{i}=1$.

We set $\mathrm{V}=\left(\mathrm{V}_{1}, \mathrm{~V}_{2}, \ldots, \mathrm{V}_{\mathrm{n}}\right)$ as an evaluation set. $\mathrm{V}_{\mathrm{j}}(\mathrm{j}=1,2, \ldots, \mathrm{n})$ represents the evaluations at all levels from high to low. When the difficult and easy level of goal and measurement is the evaluation, the evaluation set is $\mathrm{V}=$ (easy,easier,general,difficult,very difficult).

We set $F=\left(f_{1}, f_{2}, \ldots f_{n}\right)$ as a score set. It is a column vector. Among them, $f_{j}(j=1,2, \ldots, n)$ represents the score of the evaluation at the jth level. If we make the 10 score as the maximum score, we use the equal difference to give a score. So we can obtain $f_{j}=(n+1-j) * 10 / n, j=1,2, \ldots, n$,then the score set $\mathrm{F}=(10,8,6,4,2)^{\mathrm{T}}$

When we carry out the single factor evaluation for the factor set at the second level, we obtain the single factor judgment matrix $R_{i}=\left(r_{i j}^{i}\right)\left(n_{i} \times m\right)$. We have knew that various measurements have the weight of specific objective $\mathrm{g}_{\mathrm{i}}$ as $\mathrm{A}_{\mathrm{i}}=\left(\mathrm{a}_{11}, \mathrm{a}_{12}, \mathrm{a}_{13}, \ldots, \mathrm{a}_{1 \mathrm{~m}}\right)$, among them $\mathrm{a}_{\mathrm{ik}} \geq 0$, then we can calculate the fuzzy comprehensive evaluation for specific objective $g_{i}$ from $A_{i}$ and $R_{i}$, $B_{i}=A_{i} \circ R_{i},(i=1,2, \ldots, k)$.

When we carry out the evaluation and judgment on the factor at the first level, the general evaluation and judgment matrix is $R=\left(\begin{array}{ll}B_{1} & B_{2} \ldots, B_{k}\end{array}\right)^{T}$, and the comprehensive evaluation and judgment result is $A \circ R=B$.

We make use of the product of vectors to calculate the final evaluation result $\mathrm{S}$. $\mathrm{S}$ is a algebraic value, namely $S=B \circ F$.

After we calculate the $\mathrm{S}$ value of each evaluation object, we line up from big to small according to the $\mathrm{S}$ value. Then the superior sequence of all evaluation objects will be distinct.

For its optimum scheme, we also can evaluate the degree of difficulty for its implementation further. We have knew that the weight of each specific objective for general objective is $A=\left(a_{1}, a_{2}, a_{3}, \ldots a_{n}\right)$, and we obtain the fuzzy comprehensive evaluation for general objective from $A$ and $B$, namely $Z=A \circ B$. That is to say, $Z=\left[\begin{array}{lllll}Z_{1} & Z_{2} & Z_{3} & Z_{4} & Z_{5}\end{array}\right]$.

We use the fuzzy set to show it, and it is like this : $Z=Z 1 /$ easy+Z2/more easier+Z3/general+Z4/difficult+Z5/very difficult. From this we can obtain the comprehensive evaluation of B2B e-commerce company's overall development strategy in vertical industry.

By calculation we can obtain the possibility of being easy to implement the enterprise selection 
development strategy, namely 0.330. The sum of the probability for being easy for the implementation, being more easier for the implementation, and the general degree of difficulty for the implementation is 0.910 . That is to say, the probability for the strategy to get the implementation is 0.910 or so. The probability of difficulty for the implementation is 0.091 or so. Therefore, at the present the optimal strategy for enterprise development can be realized.

Theoretically we can make "whether each scheme can use the minimal resource and the minimum negative effect to achieve the consensus target" as the main basis of strategic selection. However, in the actual decision engineering, because there are some incomparable characteristics between different strategies objectively, in this way it makes the strategy scheme be selected at last so that it is influenced by various subjective and objective factors.

The realistic factors which influence the enterprise strategic selection mainly include the following things, such as the people's attitudes in the enterprise management class towards the risk, the pressure from the external environment, the influence of company culture, the business executives' personal needs and desires, the political relationship within the organization, etc. These factors will have the important influence on enterprise strategy selection in various forms. For this, when the company strategic decision makers make the strategic scheme selection, they must give the sufficient attention. At the same time, setting up the correct strategy is important actually, but the implementation of strategic scheme and the establishment of the feedback system is more important to some extent.

\section{Conclusion}

As a kind of new mode B2B e-commerce in vertical industry has nearly ten years development course. During this period, most of e-commerce companies in this industry have been accompanied by doing not so big and not so deep problems. The main reason is that each company's strategic management in this industry does not reach the designated position. Thus, we carry out the exploration of the research on the strategic scheme evaluation of B2B e-commerce in vertical industry based on AHP and fuzzy evaluation in the paper.

\section{References}

[1] Translated by Plante, Li Lulu and others. E-commerce Strategy Formulation. Beijing: China Machine Press, 2002.

[2] Dai. Q. Three Essays on B2B E-market Firms: Functionality [D]. Adoption and Alliance Strategies, Doctoral Thesis, 2003.

[3] Zha Fei and others. Translated by Wu Guanzhi. Network Marketing Strategy Implementation and Practice. Beijing: China Machine Press, 2004.

[4] Tawfik Jelassi, Albercht Enders. Translated by Li Hongxin. Strategies for E-Business Concept and Cases. Dalian: Northeast University of Finance and Economics Press, 2006.

[5] Stephen Chen. Translated by Wang Zhaoliang. Strategic Management of E-Business(the Second Edition). Beijing: Peking University Press, 2006.

[6] Turban, E. and others. Translated by Yan Jianyuan and others. E-commerce Management Perspective (the Fourth Edition). Beijing: China Machine Press, 2007.

[7] Zhao Weidong, Huang Lihua. E-commerce Mode. Shanghai: Fudan University Press, 2006.

[8] Wu Benjia. Development and Risk Aversion of E-commerce. Economic Management, 2007, 8:72-75. 
[9] Zhu Yanxu. Survival of a Chinese-style for the Websites in Vertical Industry. Business Management, 2007, 9:104-105.

[10] S.Kaplan and M.Sawhney, “The e-Hubs:the new B2B marketplaces”, Harvard Business Review, 2000, 6:97-103. 